Check for updates

Cite this: RSC Adv., 2019, 9, 17623

\title{
Microfluidic gradient device for simultaneously preparing four distinct types of microparticles $\dagger$
}

\author{
Yuanyue Liu (D)*abc
}

Preparing monodisperse, reproducible and functionally sophisticated microparticles is challenging but important for biomedical applications. Droplet microfluidics is a microparticle generation method that can precisely control the geometry and composition of microparticles. Droplet microfluidics generally produces only one type of microparticle at a time. Here, we report a simple and controllable method to simultaneously prepare four distinct types of microparticles by combining droplet generation with a gradient generator. The method can be more widely applied and with higher productivity than other microparticle generation methods due to the integration of dispersed phases which paves the way for the application to regenerative medicine. Different sizes, heterogenous and anisotropic microparticles are generated by manipulating the poly(lactic-co-glycolic acid) concentration gradient, the poly( $\varepsilon^{-}$ caprolactone)/poly(lactic-co-glycolic acid) ratio gradient, and the dimethyl carbonate/dichloromethane ratio gradient. This straightforward preparation of microparticles will promote their application in drug delivery agents, identifiers for biological assays, microsensors and tissue engineering.

Received 28th March 2019 Accepted 29th May 2019

DOI: 10.1039/c9ra02330e

rsc.li/rsc-advances part. The pH-responsive polymer poly(butyl methacrylate-co-(2dimethylaminoethyl) methacrylate-co-methyl methacrylate) (p(BMA-co-DAMA-co-MMA)) has been used for other part. Coreshell (droplet-in-droplet or a double-emulsion droplet, PLGA-rich core and the p(BMA-co-DAMA-co-MMA)-rich shell), core-double shell ( $\mathrm{p}$ (BMA-co-DAMA-co-MMA)-rich core and double shells of the PCL-rich inner layer and p(BMA-co-DAMA-co-MMA)-rich outer layer), multicore-shell (many small PLGA-rich domains embedded in a $\mathrm{p}$ (BMA-co-DAMA-co-MMA)-rich matrix), inverted core-shell (p(BMA-co-DAMA-co-MMA)-rich core and PCL-rich shell, p(BMA-coDAMA-co-MMA)-rich shell and PLGA-rich core) and Janus microparticles have been obtained by adjusting the $\mathrm{pH}$ of the continuous phase, the polymers and the organic solvent. ${ }^{15,16}$

Recently, droplet microfluidics is one of the most powerful techniques, as it can precisely control size, components, structures and specific function of microparticles. ${ }^{17,18}$ Owing to the advancement in biomaterial technology, customized microparticles have been growth successfully. ${ }^{1}$ However, droplet microfluidics generally produces only one type of microparticle at a time. Lee et al. have presented a simple microdroplet into complex emulsion microdroplet based on the diffusion of molecules at an interface. The disperse phases (monomer and Dsolvent) and continuous phases (separation agent and C-solvent) are formed disperse microdroplets at flow-focusing. Multiple emulsions, containing double, triple and quadruple emulsions, can be prepared by varying the rate of phase separation $(40 \%$, $60 \%, 80 \%) .{ }^{19}$ The customized multiple emulsions can be generated, while multi-step preparation of disperse phases should be implemented. The complicated generation steps are necessary for the preparation of customized microparticles. Therefore,
${ }^{a}$ National Engineering Research Center for Tissue Restoration and Reconstruction,
South China University of Technology, Guangzhou 510006, P. R. China
${ }^{b}$ Key Laboratory of Biomedical Materials and Engineering of the Ministry of Education,
South China University of Technology, Guangzhou 510006, P. R. China
${ }^{c}$ School of Materials Science and Engineering, South China University of Technology,
Guangzhou 510641, P. R. China
$\dagger$ Electronic supplementary information (ESI) available: Analogy between
electronics and microfluidics, detailed process of channel design, productivity
of the microfluidic gradient device, microfluidics gradient chip with eight
outlets, water contact angles measure of PLGA/PCL. See DOI: 10.1039/c9ra02330e 
a simple method for simultaneous preparation of several concentrations of microdroplets is still demanded because microparticle is a promising carrier for drugs or other factors.

A gradient generator can generate a precise, stable, and easily controlled concentration gradient. ${ }^{20,21}$ Droplet generation and gradient generator have largely been developed independently. Few studies have integrated them to simultaneously produce multiple types of microparticles. Such a combination would improve the efficiency for simultaneously preparing multiple types of microparticles and increase their yields, which paves the way for the application to more areas.

In this study, we simultaneously generated four types of microparticles by integrating droplet generation and a gradient generator. The gradient generator provides a PLGA concentration gradient, a PCL/PLGA ratio gradient, and a dimethyl carbonate (DMC)/dichloromethane (DCM) ratio gradient. Microparticles with different sizes are produced by manipulating the PLGA concentration gradient. This device enables one device to produce microspheres of different sizes at the same time. Heterogenous microparticles are produced by controlling the PCL/PLGA ratio gradient. This device realizes that it can simultaneously adjust the composition ratio of four microspheres. Anisotropic microparticles are produced by controlling the DMC/DCM ratio gradient. This device can simultaneously produce four structures of microspheres. The preparation of complex microparticles will promote their potential as drug delivery carrier, identifiers for biological assays, microsensors.

\section{Experimental}

\section{Materials}

PCL $\left(M_{\mathrm{w}}=30 \mathrm{kDa} / 40 \mathrm{kDa}\right)$ and PLGA (lactide/glycolide ratio = $\left.50 / 50, M_{\mathrm{w}}=30 \mathrm{kDa} / 40 \mathrm{kDa}\right)$ were obtained from Shandong Daigang Biomaterials (P. R. China). DCM was obtained from Tianjing Baishi Chemical Co. Ltd. (P. R. China). Rhodamine B, poly (vinyl alcohol) (PVA, 87-89\% hydrolyzed, $M_{\mathrm{w}}=88 \mathrm{kDa}$ ) and DMC were obtained from Aladdin Chemistry (Shanghai, P. R. China). Negative resist (NR21-40000P) and polydimethylsiloxane (PDMS) (Sylgard 184) were obtained from Dow Corning Company (USA) and Futurrex Inc. (USA), respectively. Glycerol was obtained from Tianjin Fuyu Chemical Co. Ltd. (P. R. China). Deionized water was obtained using a Milli-Q waterpurification system and was used all experiments.

\section{Microfluidic gradient device}

The application of circuit methods to microfluidics is based on the analogous behavior of hydraulic and electric circuits (Table 1 in $\mathrm{ESI}_{\dagger}^{\dagger}$ ). Circuit analysis is a useful method for devising intricate microfluidic networks, assuming that the fluid in microchannel is incompressible with a uniform viscosity, has a steady-state flow, and is not subject to reverse mixed flow. The pressure has a uniform gradient along the channel length. The gradient generator was designed based on an electric circuit. $^{21}$ The desired output concentrations of dispersed phases were $0 \%, 30 \%, 70 \%$ and $100 \%$. Four T-junctions of droplet generation were used for produce microspheres in a stable manner.
The pattern of the microfluidic gradient device was formed in a silicon wafer using soft lithography as reported previously. ${ }^{22,23}$ In short, a negative photoresist was spin-coated on a clean silicon wafer, which was then baked for $5 \mathrm{~min}$ at $90{ }^{\circ} \mathrm{C}$ and then for a further $30 \mathrm{~min}$ at $140{ }^{\circ} \mathrm{C}$. Ultraviolet light was exposed on the resist through the mask of pattern, the exposure time is $600 \mathrm{~s}\left(30 \mathrm{~d} \mathrm{E}^{-1}\right.$, photoresist height $d=200 \mu \mathrm{m}$, the sensitivity of photoresist $30 \mathrm{~mJ} \mathrm{~cm}{ }^{-2}$, intensity of light $E=10$ $\mathrm{mW} \mathrm{cm}{ }^{-2}$ ), then baked for $3 \mathrm{~min}$ at $100{ }^{\circ} \mathrm{C}$, cooling $30 \mathrm{~min}$ at room temperature. The resist was then developed in RD6 developer liquid. The silicon wafer with the microfluidic gradient pattern was obtained. A mixture of curing agent and PDMS $(1: 10, \mathrm{w} / \mathrm{w})$ was poured onto the silicon wafer and was allowed to solidify at $60{ }^{\circ} \mathrm{C}$ for $4 \mathrm{~h}$. The PDMS mold with microchannel was peeled off from the silicon wafer. The tailored PDMS mold was adhered to a glass slide using $\mathrm{O}_{2}$ plasma. The width and depth of all channels were $200 \mu \mathrm{m}$. To increase the hydrophilicity, the T-junction was modified by pumping through PVA/glycerol (2/5 wt\%) aqueous solution.

To verify the performance of the designed gradient generator, simulation of fluid flow in channel was performed using the COMSOL program.

\section{Synthesis of different size microparticles}

The two dispersed phases were $5 \mathrm{mg} \mathrm{ml}^{-1}$ PLGA in DMC and $40 \mathrm{mg} \mathrm{ml}^{-1}$ PLGA in DMC. The continuous phase and collecting liquid were 2 wt $\%$ PVA aqueous solutions. Two dispersed phases and four continuous phases were pumped into the microfluidic gradient device channels using syringe pumps (ColeParmer, USA and Longer, P. R. China). The flow rate of dispersed phase was $0.5 \mathrm{ml} \mathrm{h}^{-1}$. By adjusting the flow rate of the continuous phase, microspheres were simultaneously generated at four T-junctions. Then, four types of microspheres were separately collected in $2 \mathrm{wt} \%$ PVA aqueous solutions. After the volatilization of organic solvent DMC at room temperature for $24 \mathrm{~h}$, the microparticles were washed with water three times and then being freeze-dried $12 \mathrm{~h}$ (Lyophilizer, VIRTIS, USA).

\section{Synthesis of heterogenous microparticles}

The two dispersed phases were $40 \mathrm{mg} \mathrm{ml}^{-1}$ PCL in DMC and $40 \mathrm{mg} \mathrm{ml}^{-1}$ PLGA in DMC. The continuous phase and collecting liquid were $2 \mathrm{wt} \%$ PVA aqueous solutions. The procedure was carried out as described in section synthesis of different size microparticles. Due to the high solubility of DMC in water, Rhodamine B can be directly put into the collection pool of microdroplets. Microspheres were stained with Rhodamine B. The collection process needs to be protected from light to prevent fluorescence quenching. After the volatilization of organic solvent DMC, microparticles were washed with water three times and then observed by microscope.

\section{Synthesis of anisotropic microparticles}

The first dispersed phase was $40 \mathrm{mg} \mathrm{ml}^{-1}$ PCL/PLGA (5/5) in DMC. The second dispersed phase was $40 \mathrm{mg} \mathrm{ml}^{-1}$ PCL/PLGA (5/5) in a $1: 9$ DMC/DCM mixture (DMC: DCM = $1: 9$ ). Owing to the incompatible of DCM in water, Rhodamine B can 
A

$\mathrm{C}$ device.
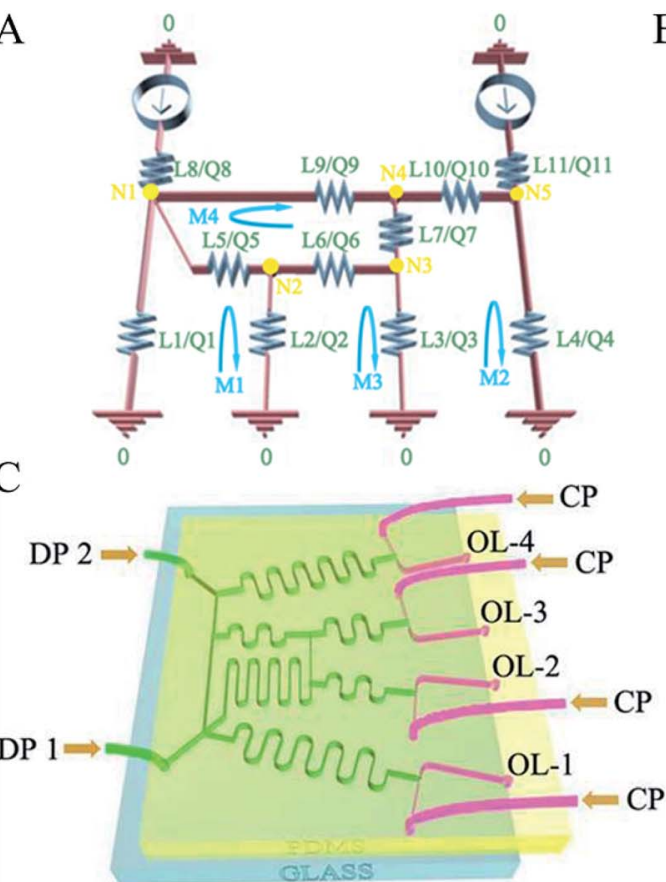

B

$\mathrm{D}$
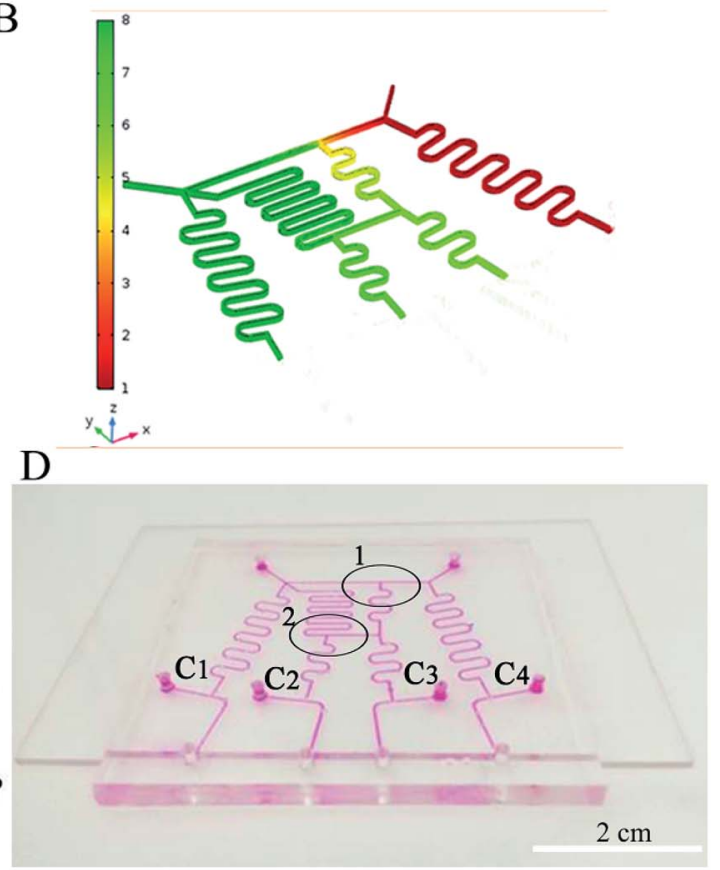

Fig. 1 Circuit diagram of the gradient generator (A), fluid simulation (B), schematic (C) and digital photograph (D) of the microfluidic gradient

be put into dispersed phase. This collection process also needs to be protected from light. The procedure was carried out as described in section synthesis of heterogenous microparticles.

\section{Characterization of microparticles}

The entire process of preparing the microparticles was monitored using an optical microscope (Dmil1, Leica, P. R. China). A confocal laser scanning microscope (CLSM) equipped with a $1 \mathrm{~mW}$ heliumneon laser (CLSM, ZeissLsm-510, Japan) was used to observe microparticles which had been stained with Rhodamine B. The freeze-dried microparticles were observed by scanning electron microscopy (SEM, FEI Quanta 200, Netherlands). Attenuated total reflection Fourier-transform infrared (ATR-FTIR) spectra (Nexus Por Euro, USA) were recorded for four types of microparticles. X-ray diffraction (XRD) patterns (X'Pert PRO, PANalytical, Netherlands) were recorded for the microparticles in the $2 \theta$ range of $10^{\circ}$ to $70^{\circ}$. Image J software was used to measure the diameters of microparticles and calculate the average microparticle diameter.

\section{Results and discussion}

\section{Microfluidic gradient device}

To simultaneously obtain multiple concentrations of microdroplets, the device was designed based on electric circuit
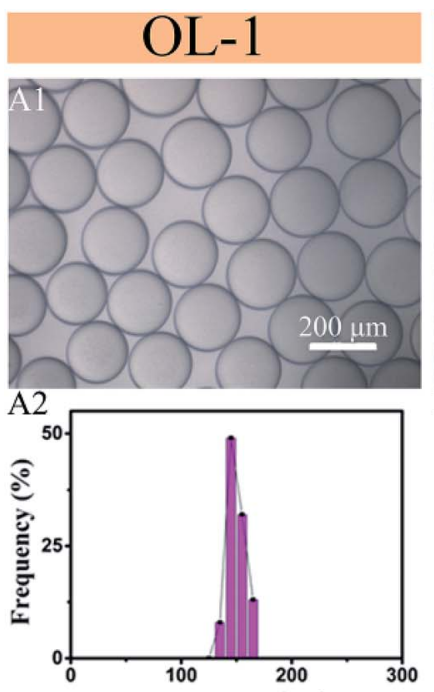
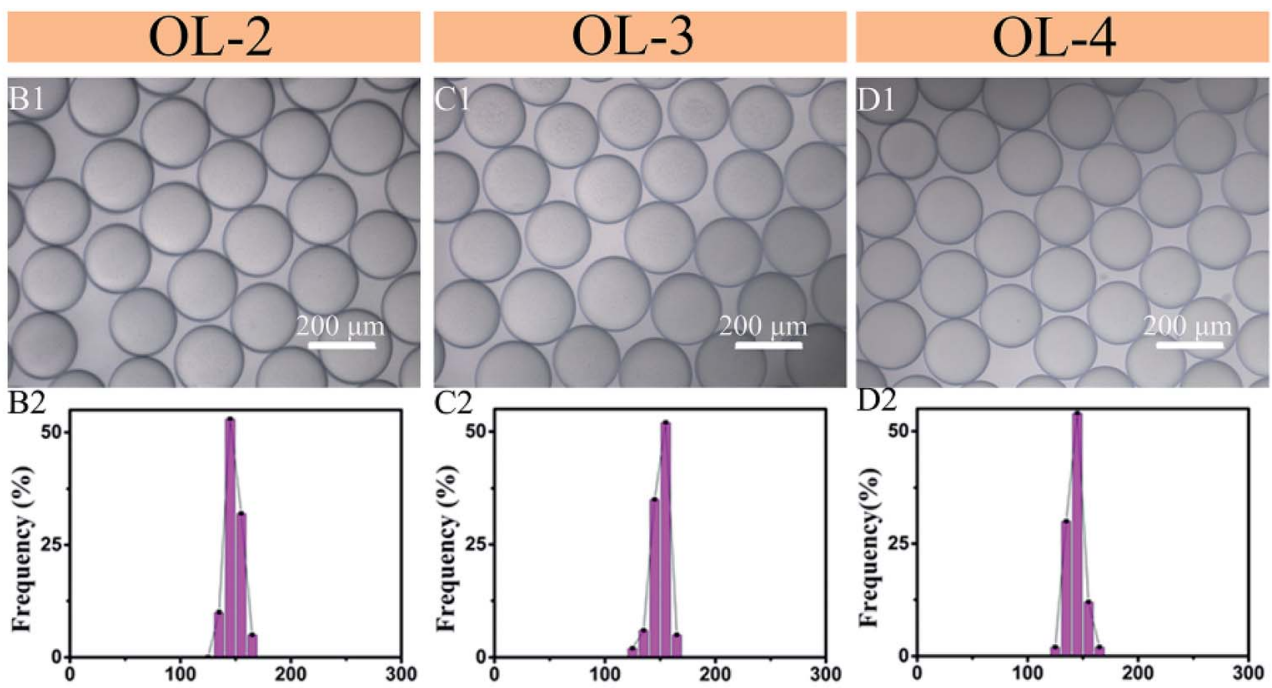

Fig. 2 Optical micrographs of microdroplets from the four outlets (A1-D1), and corresponding microdroplet distributions (A2-D2). 
methods. ${ }^{20,21}$ According to this relationship, the circuit diagram of the gradient generator with four outlets is shown in Fig. 1(A). The fluid forms a new concentration of solution by stepwise dilution in the channel. ${ }^{21}$ Four desired output concentrations $\left(C_{1}=0 \%, C_{2}=30 \%, C_{3}=70 \%\right.$ and $C_{4}=100 \%$ ) can be obtained with the device, when DP1 is $0 \%$ and DP2 is $100 \%$. The concentration of fluid in each channel is $C_{9}, C_{8}, C_{5}, C_{1}=0, C_{11}$, $C_{10}, C_{4}=100 \%, C_{7}, C_{6}, C_{3}=70 \%$ and $C_{2}=30 \%$. The detailed methods are provided in the ESI. $\dagger$ To verify the gradient module, the simulation software (COMSOL) was used to simulate the movement of fluid in microchannel. Fig. 1(B) shows the colour changing from green to red. The colour indicates a change in the concentration of fluid. It also displays that the gradient generator can produce a concentration gradient solution, and the feasibility of the designed gradient generator. Droplet generation was used to produce microdroplets. The oil phase (DP) forms microspheres under the shearing force of the incompatible aqueous phase (CP). Schematic image was shown in Fig. 1(C). The device has two dispersed phase inlets, four continuous phase inlets and four outlets. The desired concentration solution is produced by controlling the mixing ratio of the two combined solutions. A new solution concentration $\left(C_{3}\right.$ $=70 \%)$ forms in the first mixing of dispersed phase 1 (DP1) $\left(C_{1}\right.$
$=0 \%)$ and dispersed phase 2 (DP2) $\left(C_{4}=100 \%\right)$. The second mixing of $C_{1}$ and $C_{3}$ forms another new solution concentration $\left(C_{2}=30 \%\right)$ (Fig. 1(D)). Four different concentrations of solution are generated by the gradient generator. Four concentrations of microdroplets are simultaneous produced at the four $\mathrm{T}$ junctions. This device enables the possibility of simultaneously preparing multiple concentrations of microspheres. In comparision with other device with one type droplets at a time, it can reduce experimental steps, save time and increase the efficiency of producing microdroplets. ${ }^{12,19,24,25}$

The four types of microdroplets are shown in Fig. 2(A1-D1). This device improves the yield of microparticles. According to the design, the flow rates of four dispersed phases before forming the microspheres are the same. The diameters of the four kinds of droplets are similar because of the same formation device and velocity of continuous phase. Each type of microdroplet has a uniform size distribution, as shown in Fig. 2(A2-D2). The diameter of the uncured microdroplet is about $150 \mu \mathrm{m}$ at four outlets. To further illustrate, the use of microfluidic gradient device can increase the yield of the microspheres. We also designed a microfluidic gradient device with eight outlets, and the concentrations of microspheres were exponentially distributed, as shown in the ESI. $\dagger$
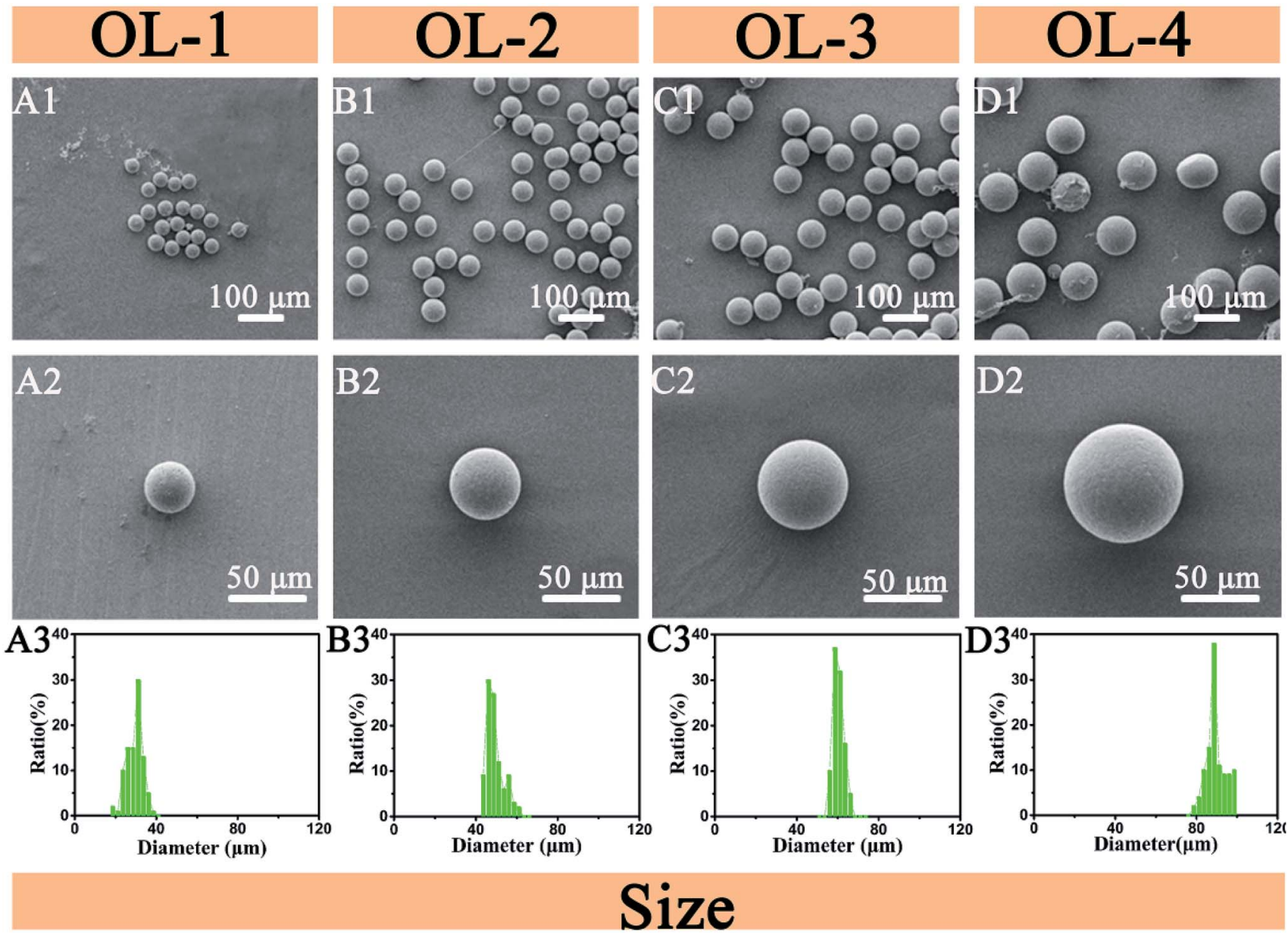

Fig. 3 SEM images (A1-D1 and A2-D2) and size distributions (A3-D3) of the four sizes of PLGA microparticles. 


\section{Different size microparticles}

The two dispersed phases PLGA/DMC (DP1 $=5 \mathrm{mg} \mathrm{ml}^{-1}$, $\mathrm{DP} 2=40 \mathrm{mg} \mathrm{m} \mathrm{m}^{-1}$ ) were pumped into the microfluidic gradient device to form PLGA gradient concentration solutions. These solutions then formed four concentrations of microdroplets at the T-junctions. After the volatilization of DMC, four different concentrations of microdroplets are solidified into different PLGA particle sizes, ${ }^{26}$ as shown in Fig. 3(A2-D2). The concentration of solute in microdroplet is different, resulting in different sizes of microparticles. The microparticles of each size have narrow size distributions, as shown in Fig. 3(A1-D1 and A3-D3). The average diameters of the four types of microparticles are $29.95 \mu \mathrm{m}$, $49.35 \mu \mathrm{m}, 60.4 \mu \mathrm{m}$, and $86.075 \mu \mathrm{m}$. The formed PLGA microparticles have a smooth surface. With the increase of the concentration, the particle size gradually increased. By controlling the concentration of PLGA, the device achieves the regulation of microparticles size. When the microsphere is used as a drug carrier, it can simultaneous regulate the loading of drugs.

\section{Heterogenous microparticles}

Heterogenous microparticles with different PCL/PLGA ratios are shown in Fig. 4. The two dispersed phases (PCL/DMC $=$ DP1, PLGA/DMC = DP2) were injected into the microfluidic gradient device to form different PCL/PLGA ratios of solution. The volatilization of DMC causes the microdroplets containing pure PCL or pure PLGA to form into rough and smooth spherical structures, respectively, as shown in Fig. 4(A1 and D1). The microdroplets containing different ratios of PCL/PLGA gradually segregate. Boundaries formed at the surface of microparticles, resulting in the Janus structure shown in Fig. 4(B1 and C1). Increasing the PLGA/PCL mass ratio causes the dividing line to gradually move towards the rough compartment, as shown in Fig. 4(A1-D1). This device can simultaneously produce four types, while other methods about heterogenous microparticles need four experiments and more time and energy. ${ }^{27}$

To confirm this structure, Rhodamine B was used to stain the microparticles. Fig. 4(A2-D2) shows that Rhodamine B selectively stains PLGA, leaving the PCL region of the microparticles unstained. This indicates the separation of two polymers in the microparticles. By measuring the contact angles of
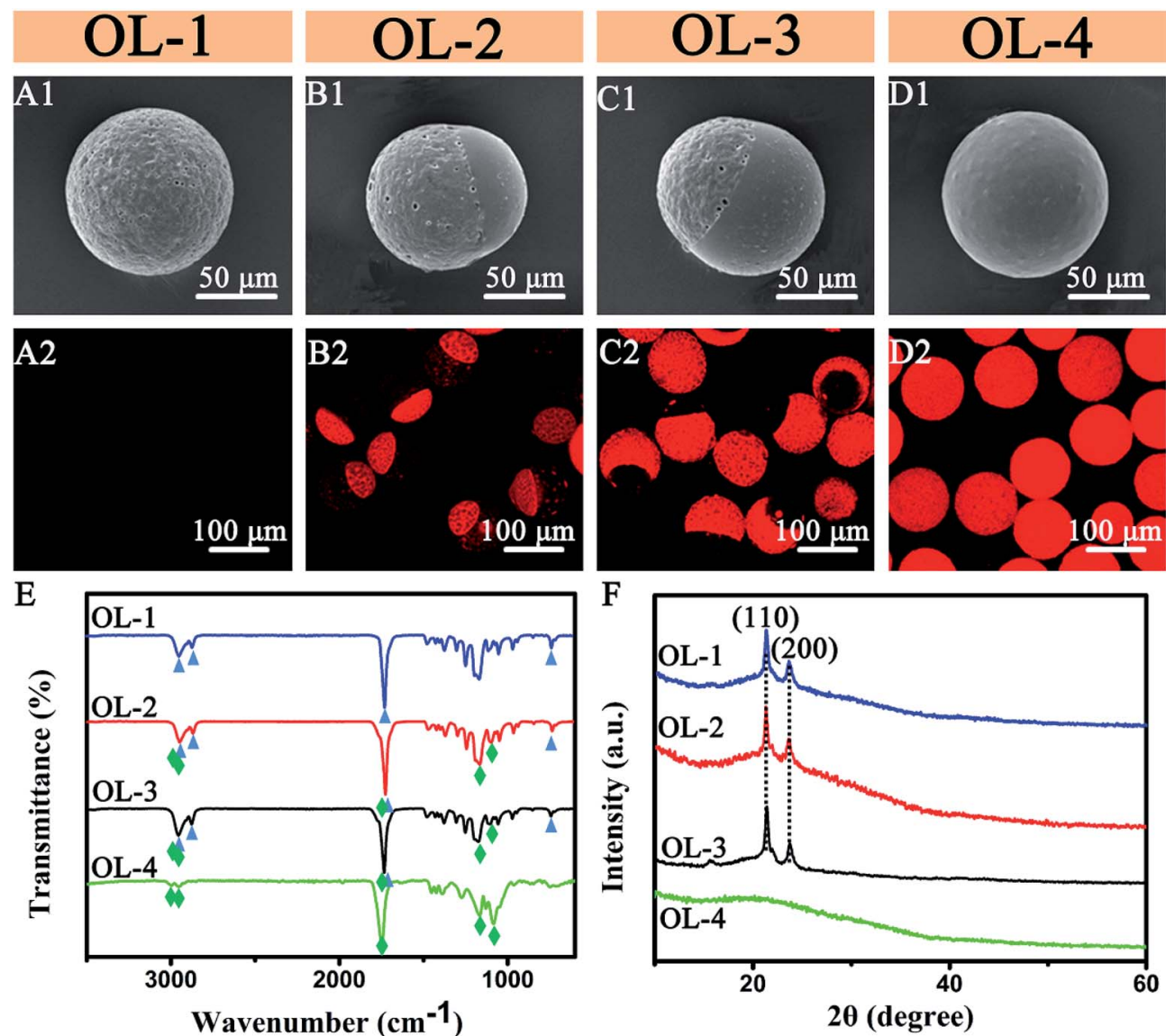

\section{Heterogeneity}

Fig. 4 SEM images (A1-D1), CLSM images (A2-D2), ATR-FTIR spectra (E) and XRD patterns (F) of the heterogenous microparticles. (A) PCL, (B) $P C L: P L G A=7: 3$, (C) PCL : PLGA = 3: 7, (D) PLGA. 
PLGA and PCL, it is found that PLGA is wetting and PCL is nonwetted (ESI $\dagger$ ). Rhodamine $\mathrm{B}$ is a dye soluble in water. This explains why Rhodamine B can selectively dye PLGA materials. To figure out the distribution of two materials on the microparticle surface, ATR-FTIR spectra and XRD patterns of the four types of microparticles are shown in Fig. 4(E and F). The absorption peaks of PCL (curve OL-1) at 2946, 2865, 1737 and $731 \mathrm{~cm}^{-1}$ and PLGA (curve OL-4) at 2998, 2951, 1759, 1129 and $1086 \mathrm{~cm}^{-1}$ are observed in the ATR-FTIR spectrum of the Janus particles (curve OL-2, OL-3). This phenomenon displays that Janus particles have two materials. It also confirms that the Janus microparticles are composed of PLGA and PCL, and the four types of microparticles are heterogeneous. Fig. 4(F) shows the XRD patterns of microparticles. In the XRD patterns of OL-1, OL- 2 and OL-3, peaks at $2 \theta$ of $21.5^{\circ}$ and $23.8^{\circ}$ correspond to the (110) and (200) planes, respectively. The microparticles from the OL-1, OL-2 and OL-3 contain semi-crystalline PCL polymer components. No obvious diffraction peaks are observed in the XRD pattern of amorphous polymer PLGA (OL-4) in Fig. 4(F). In summary, the XRD results also confirm that four types of microparticles are heterogeneous.

Phase separation mainly depends on interfacial tension and diffusion coefficient between immiscible phases and these interactions. ${ }^{28}$ These determinants are influenced by many factors. Some aspects of the interfacial tension and diffusion coefficient (such as the temperature, PLGA/PCL ratio, DMC/ DMC rate) between the different phases may result in dewetting and wetting, as reported previously. ${ }^{27}$ Therefore, complex microspheres can be obtained by changing the parameters of the ratio. Here, PCL and PLGA, biomaterials with biocompatibility, hydrophobility/hydrophilicity and biodegradability, are chosen as model materials. Solvent volatilization phase separation is the dissolution of two immiscible polymers in the same volatile organic solvent. After the organic solvent is volatilized, the two polymers begin to phase separate and occupy their respective rich phase to form Janus particles. The Janus structure can be considered using the partial wetting theory and classic spreading: $S_{\mathrm{i}}=r_{\mathrm{jk}}-\left(r_{\mathrm{ij}}+r_{\mathrm{ik}}\right)$. In this part, the different ratio of PLGA/PCL changes the interfacial tension between the different phases and the spreading coefficients. We define the oil phase including PLGA, $2 \mathrm{wt} \%$ PVA aqueous solutions and the oil phase including PCL as 1,2 and 3. DMC has much higher solubility in water $\left(13.9 \mathrm{wt} \%\right.$ at $\left.20^{\circ} \mathrm{C}\right)$ and then quickly can be extracted from the microdroplet $(<30 \mathrm{~s}) .{ }^{29}$ According to the theory, $S_{1}=r_{23}-\left(r_{12}+r_{13}\right), S_{2}=r_{13}-\left(r_{12}+r_{23}\right), S_{3}=r_{12}-\left(r_{13}+\right.$ $\left.r_{23}\right)$. The interfacial tension between PLGA and PCL in DMC can be obtained from their surface energy. According to the contact angles of PLGA and PCL, the results is that $r_{13}>r_{23}$, and then $S_{1}$ $<0$. Owing to high solubility in water of DMC, the interfacial tension of $r_{12}$ is small. The value is that $S_{2}>0, S_{3}<0$. The spreading coefficient $S_{2}$ is positive. $S_{1}$ and $S_{3}$ are negative, which satisfies the condition of dewetting. ${ }^{29}$ This will present Janus dewetting structure.

\section{Anisotropic microparticles}

SEM and CLSM images of the anisotropic microparticles are shown in Fig. 5. The two dispersed phases (DP1, DP2) were poured into the microfluidic gradient device to form different
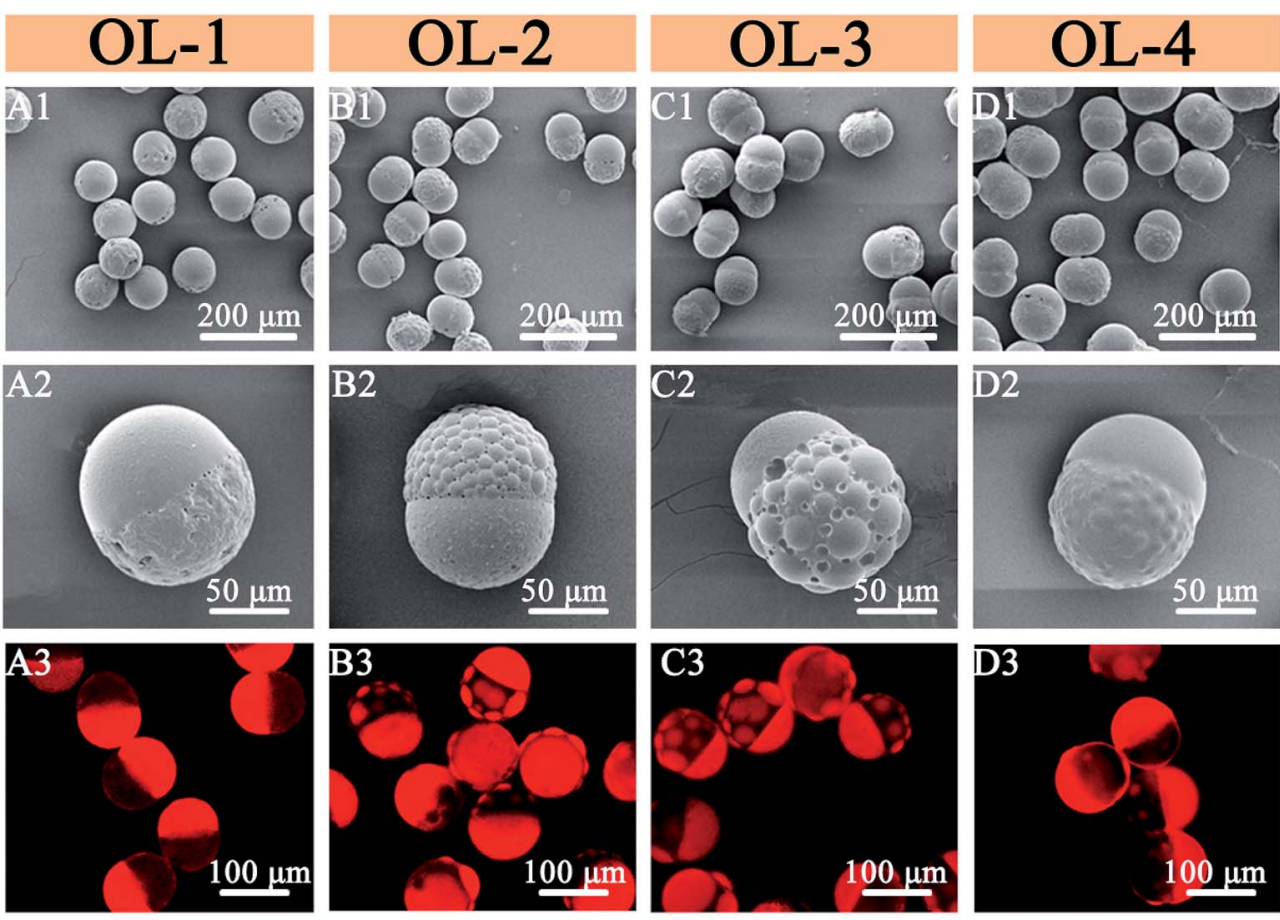

\section{Anisotropy}

Fig. 5 SEM images (A1-D1 and A2-D2) and CLSM images (A3-D3) of anisotropic microparticles 
DMC/DCM ratios of solution. After the organic solvent evaporated, four different structures will appear. SEM images of the uniform microparticles are shown in Fig. 5(A1-D1). The Janus particles in Fig. 5(A2) have an interface at their centers. The three types of patchy Janus particles are composed of a smooth region and a sea-island region, as shown in Fig. 5(B2-D2). The four types of microparticles are anisotropic. CLSM images of the microparticles dyed with Rhodamine B are shown in Fig. 5(A3D3). The patchy Janus particles are stained in one region and the other partial region, showing the distribution of PLGA in the anisotropic microparticles and confirming that the surface patches are PLGA materials.

The different ratios of DMC/DCM affect the interfacial tension between the different phases and also affect the spreading coefficients. The partial wetting theory and classic spreading, $S_{\mathrm{i}}=r_{\mathrm{jk}}-\left(r_{\mathrm{ij}}+r_{\mathrm{ik}}\right)$, can also explain the Janus patchy formation mechanism. ${ }^{29}$ The organic solvent is composed of different DMC/DCM ratios. The solubility of DCM in water is low $\left(2 \mathrm{wt} \%\right.$ at $\left.20{ }^{\circ} \mathrm{C}\right) .{ }^{29}$ The solution of PLGA and PCL in DMC or its mixture with DCM are completely mixed for some time. The interfacial tension $r_{13}$ is considered as zero. The interfacial tension of $r_{12}$ exceeds $r_{13}$, owing to the hydrophilicity of PLGA and the hydrophobility of DCM and PCL. This results is that $S_{1}<0, S_{2}<0, S_{3}>0$, which is for patchy Janus structure. Owing to the existence of DMC (high solubility in water), phase separation between PLGA and PCL in DMC/DCM solution is same as in pure DMC at the beginning. Subsequently, phase separation is same as in pure DCM. $^{29}$ The former results in the formation of the Janus structure, whereas the latter causes the patchy structure, which finally forms the patchy Janus structure. With the increase of DCM/DMC ratio, three different degrees of patchy Janus wetting structure are appeared.

\section{Conclusions}

In summary, this work developed the simultaneous preparation of four types of microparticles by integrating droplet generation with a gradient generator. Microparticles with different size are formed as a result of the PLGA concentration gradient. Heterogenous microparticles are formed as a result of the PCL/PLGA ratio gradient. Anisotropic microparticles are formed as a result of the DMC/DCM ratio gradient. The gradient generator precisely controls the gradient solution (i.e. PLGA, PLGA/PCL, and DMC/DCM concentration gradients), and droplet generation produces the various microparticles (i.e. different sizes, heterogenous, anisotropic). The preparation of complex microparticles by the microfluidic gradient device may facilitate the application of microparticles in drug delivery agents, active pigments for displays, microsensors, and interface stabilizers.

\section{Conflicts of interest}

There are no conflicts to declare.

\section{Acknowledgements}

This work was supported by Laboratory \& Facility Management Division of South China University of Technology and Ashleigh Cooper, PhD, from Liwen Bianji, Edanz Editing China (www.liwenbianji.cn/ac), for editing the English text of a draft of this manuscript.

\section{References}

1 W. Li, L. Y. Zhang, X. H. Ge, B. Y. Xu, W. X. Zhang, L. L. Qu, C. H. Choi, J. H. Xu, A. Zhang, H. M. Lee and D. A. Weitz, Chem. Soc. Rev., 2018, 47, 5646-5683.

2 A. Choi, K. D. Seo, D. W. Kim, B. C. Kim and D. S. Kim, Lab Chip, 2017, 17, 591-613.

3 T. Nisisako, Curr. Opin. Colloid Interface Sci., 2016, 25, 1-12. 4 B. Wang, P. Prinsen, H. Wang, Z. Bai, H. Wang, R. Luque and J. Xuan, Chem. Soc. Rev., 2017, 46, 855-914.

5 D. Liu, H. Zhang, F. Fontana, J. T. Hirvonen and H. A. Santos, Lab Chip, 2017, 17, 1856-1883.

6 T. Y. Lee, T. M. Choi, T. S. Shim, R. A. Frijns and S. H. Kim, Lab Chip, 2016, 16, 3415-3440.

7 J. H. Kim, T. Y. Jeon, T. M. Choi, T. S. Shim, S. H. Kim and S. M. Yang, Langmuir, 2014, 30, 1473-1488.

8 C. Salvador-Morales, B. Brahmbhatt, V. Marquez-Miranda, I. Araya-Duran, J. Canan, F. Gonzalez-Nilo, C. Vilos, J. Cebral, F. Mut, R. Lohner, B. Leong, G. Sundaresan and J. Zweit, Langmuir, 2016, 32, 7929-7942.

9 J. Leijten, J. Seo, K. Yue, G. T. Santiago, A. Tamayol, G. U. Ruiz-Esparza, S. R. Shin, R. Sharifi, I. Noshadi, M. M. Alvarez, Y. S. Zhang and A. Khademhosseini, Mater. Sci. Eng., R, 2017, 119, 1-35.

10 X. Pang, C. Wan, M. Wang and Z. Lin, Angew. Chem., Int. Ed., 2014, 53, 5524-5538.

11 Q. Wu, C. Yang, G. Liu, W. Xu, Z. Zhu, T. Si and R. X. Xu, Lab Chip, 2017, 17, 3168-3175.

12 W. Shi and D. A. Weitz, Macromolecules, 2017, 50, 7681-7686.

13 R. Ran, Q. Sun, T. Baby, D. Wibowo, A. P. J. Middelberg and C.-X. Zhao, Chem. Eng. Sci., 2017, 169, 78-96.

14 C.-H. Choi, S.-M. Kang, S. H. Jin, H. Yi and C.-S. Lee, Langmuir, 2015, 31, 1328-1335.

15 N. G. Min, B. Kim, T. Y. Lee, D. Kim, D. C. Lee and S. H. Kim, Langmuir, 2015, 31, 937-943.

16 N. G. Min, M. Ku, J. Yang and S.-H. Kim, Chem. Mater., 2016, 28, 1430-1438.

17 J. Wang, Y. Li, X. Wang, J. Wang, H. Tian, P. Zhao, Y. Tian, Y. Gu, L. Wang and C. Wang, Micromachines, 2017, 8, 22.

18 L. Shang, Y. Cheng and Y. Zhao, Chem. Rev., 2017, 117, 79648040.

19 C. H. Choi, D. A. Weitz and C. S. Lee, Adv. Mater., 2013, 25, 2536-2541.

20 K. Lee, C. Kim, B. Ahn, R. Panchapakesan, A. R. Full, L. Nordee, J. Y. Kang and K. W. Oh, Lab Chip, 2009, 9, 709717.

21 K. W. Oh, K. Lee, B. Ahn and E. P. Furlani, Lab Chip, 2012, 12, 515-545. 
22 T. Ma, X. Gao, H. Dong, H. He and X. Cao, Appl. Mater. Today, 2017, 9, 49-59.

23 Y. Fan, X. Cao, T. Hu, X. Lin, H. Dong and X. Zou, J. Phys. Chem. C, 2016, 120, 3955-3963.

24 E. E. Ekanem, Z. Zhang and G. T. Vladisavljevic, J. Colloid Interface Sci., 2017, 498, 387-394.

25 R. Deng, H. Li, J. Zhu, B. Li, F. Liang, F. Jia, X. Qu and Z. Yang, Macromolecules, 2016, 49, 1362-1368.
26 J. Du and R. K. O'Reilly, Chem. Soc. Rev., 2011, 40, 2402-2416. 27 W. Li, H. Dong, G. Tang, T. Ma and X. Cao, RSC Adv., 2015, 5, 23181-23188.

28 T. Tanaka, R. Nakatsuru, Y. Kagari, N. Saito and M. Okubo, Langmuir, 2008, 24, 12267-12271.

29 X. D. Cao, W. X. Li, T. Ma and H. Dong, RSC Adv., 2015, 5, 79969-79975. 